\title{
INFLUENCE OF STERILIZATION OF A PRODUCT MANUFACTURED USING FDM TECHNOLOGY ON ITS DIMENSIONAL ACCURACY
}

\author{
Wiesław Kuczko', Radosław Wichniarek', Filip Górski', Jacek Banaszewski² \\ 1 Poznan University of Technology, Chair of Production Engineering and Management, Poznan, Poland, \\ e-mail: wieslaw.kuczko@put.poznan.pl, radoslaw.wichniarek@put.poznan.pl, filip.gorski@put.poznan.pl \\ 2 Poznan University of Medical Sciences, Department of Otolaryngology Head and Neck Surgery, Poznan, \\ Poland, e-mail: jbanasz@ump.edu.pl
}

Received: 2017.10 .26

Accepted: 2018.02.01

Published: 2018.03.01

\begin{abstract}
Inter-operational surgery supplies manufactured additively using the FDM (Fused Deposition Modelling) technology are required to be sterilized before use. After manufacturing, the part should be sterilized using one of commonly used processes, without losing its dimensions and shape. The paper presents studies on manufacturing and sterilizing samples made out of ABS material and influence of the sterilization process on the dimensional accuracy of these samples.
\end{abstract}

Keywords: medical, additive manufacturing, Fused Deposition Modelling, geometric accuracy

\section{INTRODUCTION}

Additive manufacturing (also known as 3D printing) is already a well-known and popular method of obtaining products. Layered character of manufacturing allows obtaining products of very complex geometry, which would be very costly or even impossible to achieve with machining. It is the most beneficial to use additive manufacturing in piece or small series production [4].

Thanks to possibility of manufacturing complex, organic shapes, the method is very much suitable for medical applications. Accurate physical representation of human anatomical shapes is one of the most demanding tasks of manufacturing technologies supplying doctors of medicine. Due to advantages of medical products and tools adjusted directly to a particular patient, development of additive manufacturing technologies in medicine is very dynamic [12].

Limitations of use of additive manufacturing in medicine are caused mostly by the range of available and verified materials, having appropriate certificates [2]. These materials, just as devices for their processing are expensive in comparison to other widespread and available materials for additive manufacturing. The most strict limitations apply to invasive medical procedures, where biocompatibility and sterility of a product in contact with patient's body is a basic problem [7]. Not each medical product requires long contact with human body, which allows to give up the first of mentioned requirements in certain cases, with sterility remaining a problem to overcome. Nowadays, there are many methods of product sterilization, which in context of this work should not only guarantee sterility of a product but also allow physical and chemical properties to remain intact.

Among many additive manufacturing methods and available technologies, Fused Deposition Modelling (FDM) is the most widespread process [5]. It results from the following factors: relatively small prices of machines, wide selection of materials with different physical properties and relatively good accuracy of obtained products $[3,6]$. The method is used, among other things, in such branches as: automotive, aeronau- 
tics and machine industry. Also in medicine it is a method which finds more and more applications, thanks to its advantages, for example in manufacturing of personalized orthopedic supplies or low-cost limb prostheses. Among many possible thermoplastic materials, the most popular are ABS (Acrylonitrile-butadiene-styrene) and PLA (Polylactic acid) [10].

The authors (cooperation between a technical university and a clinic of medical university) conducted studies aimed at confirmation of possibilities of manufacturing and use of 3D printed ABS products in surgery rooms. The products are templates for lower jaw reconstructive surgery with the use of free flaps from patient's own fibula or tibia bone. First stage of these studies, presented in this paper, was to verify possibilities of sterilization of products manufactured at the technical university, using sterilization methods available at the clinic.

\section{THEORETICAL INFORMATION}

Nowadays, there are five areas in which technologies of additive manufacturing can bring the best support for medicine [13]:

- medical models - for needs of educational purposes, allowing discussion and mutual understanding between doctors and patients,

- medical supplies used during therapy or rehabilitation - e.g. orthoses of ankle joint, shoe insets, hand prostheses,

- inter-operational surgery supplies - templates and measures for cutting, drilling and positioning,

- implants,

- organs and tissues (biomanufacturing).

Additively manufactured models and interoperational surgery supplies are usually elements of small dimensions. Their geometry is designed directly on the basis of patient anthropometric data, gathered before the operation, using technologies such as Computed Tomography (CT) or other medical imaging techniques. In a prevalent amount of cases, they are applied when a surgery is performed on patient's skeletal system.

Products of this type are usually manufactured from metals [11] or polymers [1], while the letter ones are much cheaper in most cases. Obviously, due to direct contact with patient body occurring in the surgery room, selection of an appropriate method and material for manufacturing of interoperational supplies is very significant. Just as for each product used in the operating room, aspects related to cleanliness and sterility must be considered. In case of manufacturing implants, which will remain in patient body for a longer time, it is necessary for a material and a process to be compatible with appropriate standards (namely, ISO 10993). Despite significant development of additive manufacturing technologies, only a small amount of materials and machines have appropriate certificates, which is why access to them is limited. In case of single-use products, which have limited contact with a patient, much wider range of materials and processes can be used.

The inter-operational models used by surgeons during operations are very often in contact with the patient only for several dozens of seconds or a few minutes, but their application may reduce time of the whole operation by hours. Independently on time of its use, the inter-operational model must be sterile and it is supplied into an operating room in an appropriate packing, with a description guaranteeing its use for appropriate patient [8]. Manufacturing a product in conditions, which are not sterile, sterilization should be ensured. The applied method should make the whole product sterile on the one hand, and on the other hand will not influence other technical coefficients, including dimensional and shape accuracy. Danger of not fulfilling these conditions increases along with complexity of shape of a given product.

In literature, possibility of sterilization of products made out of ABS material is confirmed $[9,10]$. Usefulness of an inter-operational supply for a doctor, evaluated by dimensional and shape accuracy, is a result of combination of four features: appropriate geometry, used material, machine processing the material and the sterilization process. In case of a lack of unequivocal data indicating possibility of use of a given combination for manufacturing of a specific type of medical supplies, it is necessary to conduct experimental trials before direct use in patient treatment.

\section{PRACTICAL STUDIES}

The tests consisted in manufacturing a number of samples using the FDM process out of ABS materials, sterilizing them and then measuring their shape and dimensions with a 3D scanner. 
Sample geometry (Fig. 1) was designed in a way to make it easier for measurement, while its size was selected to represent a shape needed in the operating room. The sample was modeled in the CATIA v5 CAD system. Then it was converted to the STL format (triangular mesh), acceptable by the FDM 3D printer software.

Production of test samples was realized on the Stratasys Dimension BST 1200 machine. As a model material, polymer ABS material was used, with producer designation $\mathrm{P} 400$. The material was black and the whole batch of samples was manufactured out of the same production series. Each sample was manufactured separately to maintain identical way of placing it in the working chamber, to avoid any differences in geometry caused by different thermal conditions.

Generation of G-code for the additive manufacturing machine was realized in the CatalystEX software. Layer thickness was $0,245 \mathrm{~mm}$, with solid $(100 \%)$ internal filling of each layer. Algorithm of support generation was selected as the preset named SMART. All the other values of manufacturing parameters were left at default

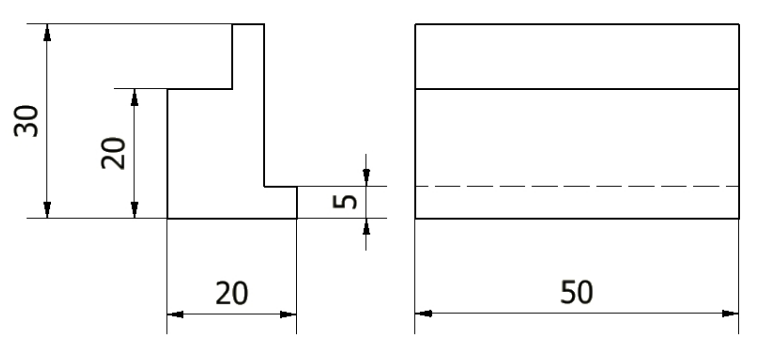

Fig. 1. Geometry of test samples values. Ready sample and its positioning on the modelling table is visible in Fig. 2.

Manufacturing of a single sample lasted 49 minutes and used $19,39 \mathrm{~cm}^{3}$ of build material and $0,9 \mathrm{~cm}^{3}$ of support material for the base layer (also known as the raft, preventing deformations of part during its removal from the table. Total 21 samples were manufactured for further studies. All the samples were manufactured correctly visual evaluation performed during manufacturing and after removing ready samples out of the working chamber did not allow stating any visible defects of samples.

At this stage, 20 samples were divided into 4 measurement series and were transported to the clinic, where each series was sterilized using different methods and conditions. One sample was left as a reference element. Two series of samples were sterilized using the STERAD 100 device, which works over the principle of low temperature plasma sterilization. In both cases, temperature of $55^{\circ} \mathrm{C}$ and pressure of $0,002 \mathrm{MPa}$ was applied, but samples of each series in the machine chamber were set differently. The first series was placed identically as in the additive manufacturing machine (horizontal orientation). The second series samples were sterilized in vertical orientation (rotation by $90^{\circ}$, long edge in vertical position). Single sterilization lasted 7 minutes. Each sample was sterilized separately and the procedure was repeated twice, maintaining a pause during which the sample returned to ambient temperature.

The second method of sterilization was a device BMT STERIVAP 669-2FD. It is an autoclave, sterilizing products using water vapor. The sterilization process lasted 7 minutes and temper-

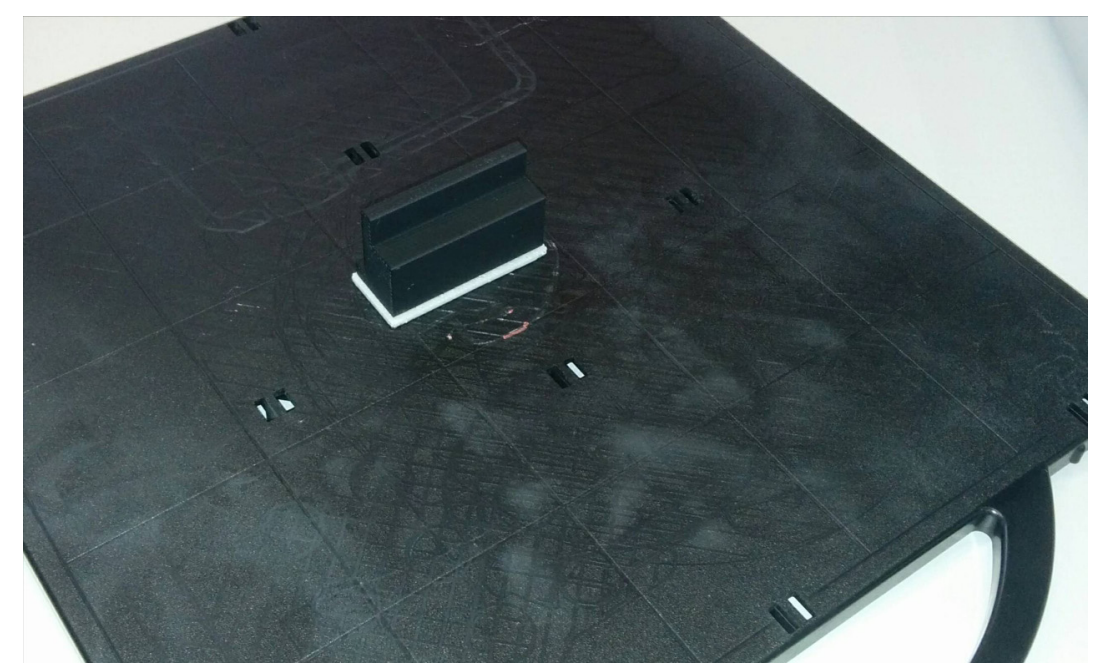

Fig. 2. Orientation of a test sample on a modelling table of the Dimension BST 1200 device 
ature inside the device was $134^{\circ} \mathrm{C}$. Pressure inside the chamber was set to $0,28 \mathrm{MPa}$. Similarly as in the first method, two series of samples were used, with different sample orientation. Each sample was sterilized in separate process.

After sterilization, each sample was hermetically packed (Fig. 3), a standard practice in clinical conditions. This ensures sterility for storage and transport into the operating room. Samples of all series in a state as in Fig. 3 were then transported to the technical university, for geometry measurements.

As a measuring device, the authors used GOM Atos Compact Scan 5M 3D scanner, working on principle of blue light, allowing non-contact measurement of objects in measurement area of $150 \mathrm{~mm}$, which was applied in the presented studies. Each samples, including the reference one, were scanned in identical ambient conditions, maintaining the same procedure. Gathered measurement data out of each sterilized sample were then compared with a reference sample. The comparison was realized during the GOM Inspect software in version 7. Mutual fitting of measurement results was made using the best-fit principle.

\section{RESULTS AND DISCUSSION}

Samples of both series sterilized in autoclave using water vapor deformed significantly. Geometrical deformations of all samples were vis- ible to a naked eye. It was expected, as the process temperature was higher than the softening temperature of the used polymer. On the other hand, scale of deformation was higher than the pre-estimates.

The series of samples sterilized by water vapor in horizontal position was characterized with average best-fit of $1,62 \mathrm{~mm}$. It is a significant deviation, which totally disqualifies this method of sterilization for this kind of products. Figure 4 shows fitting of one of samples of this series to a reference sample. For better visibility, colorful deviation map was not used, as the deviations are clearly visible. The test samples have specific deformations formed by pushing against a basket, in which they were inserted into the autoclave. As a result of deformation, the samples were lengthened from the nominal $50 \mathrm{~mm}$ to $63,4 \mathrm{~mm}$ in average (measured in the widest locations). Height of samples were decreased from nominal $30 \mathrm{~mm}$ to average of $24,9 \mathrm{~mm}$ and $26,3 \mathrm{~mm}$ on two opposite sides. Burring of the material was visible on ends of all samples.

Samples sterilized by water vapor in vertical orientation obtained average best-fit of $1,71 \mathrm{~mm}$ - similarly as in the horizontal orientation. Figure 5 presents fitting of a sample of this series to the reference sample - clear swelling of sample is visible, as opposed to the horizontal orientation sample series, here length was reduced from nominal $50 \mathrm{~mm}$ to average $43,7 \mathrm{~mm}$, at the same time width of sample increased from nominal 30

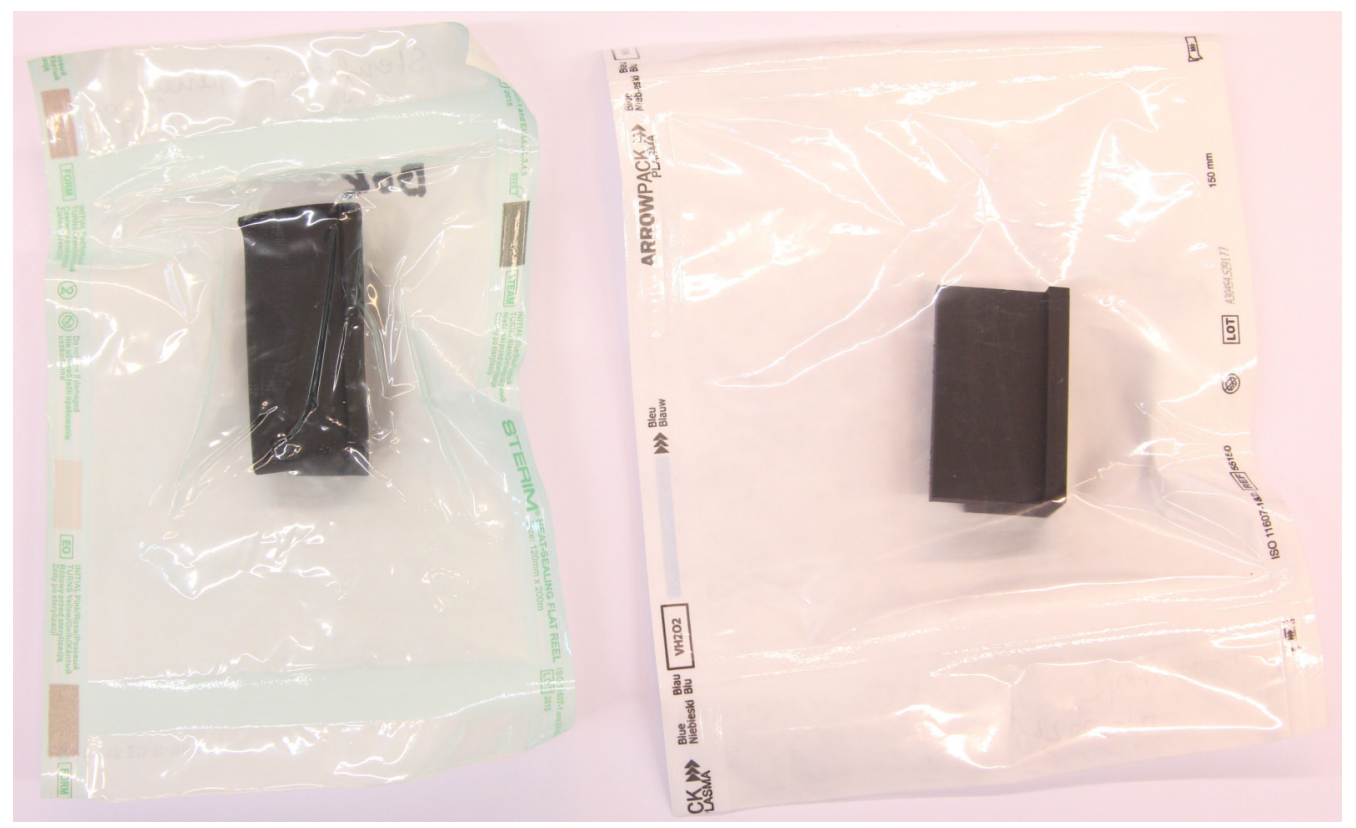

Fig. 3. Exemplary samples after sterilization, enclosed in protective packing 

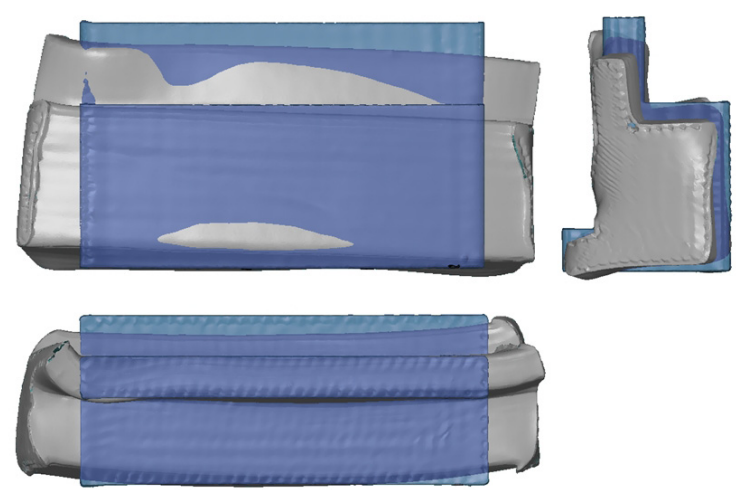

Fig. 4. Fitting of sample sterilized by water vapor in horizontal location (grey) to the reference sample (blue)

$\mathrm{mm}$ to average of $39 \mathrm{~mm}$. Additionally, thin walls of samples were significantly warped.

The plasma sterilization process brought much more beneficial results. Average best-fit for the horizontal orientation samples were 0,036 $\mathrm{mm}$. It is a much lower value than repeatability declared by the FDM machine producer, which is $0,2 \mathrm{~mm}$. The samples maintained their shape with maximal deviations of $+0,12 \mathrm{~mm}$ and $-0,07 \mathrm{~mm}$. Exemplary deviation map is presented in Figure 6.

Worse effects were obtained using plasma sterilization for vertically oriented samples - average best-fit to the reference sample was 0,106 $\mathrm{mm}$. Visual evaluation of all samples did not allow to state any deformations or other defects. Figure 7 presents colorful deviation map for the worse sample of the series. Decrease of sample height along normal axis of a plane, on which the sample was placed during the sterilization, is clearly visible - from nominal $50 \mathrm{~mm}$ to average value of $49,5 \mathrm{~mm}$. The samples maintained their shape with maximal deviations of $+0,15 \mathrm{~mm}$ and $-0,35 \mathrm{~mm}$.

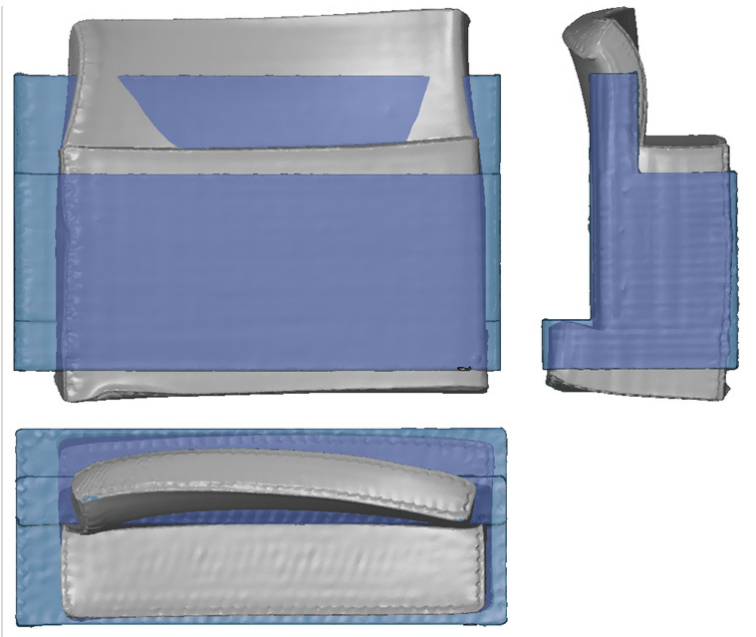

Fig. 5. Fitting of sample sterilized by water vapor in vertical location (grey) to the reference sample (blue)

\section{CONCLUSIONS}

The studies conducted by the authors allowed to state that it is possible to manufacture and sterilize inter-operational supplies made out of thermoplastic material such as ABS. The samples manufactured additively can be successfully sterilized in plasma and this process does not influence significantly their shape and dimensional accuracy. As expected, water vapor sterilization is unachievable for this kind of material, as the temperatures are too high - it could be viable for other 3D printed polymers, such as polycarbonate (PC) or polyphenylsulfone (PPSF). The developed sterilization methodology was a basis for creation of real inter-operational supplies used in surgery with success. However, it must be noted that both test samples and later inter-operational supplies were products of relatively small volumes and compact structure. Different orientation
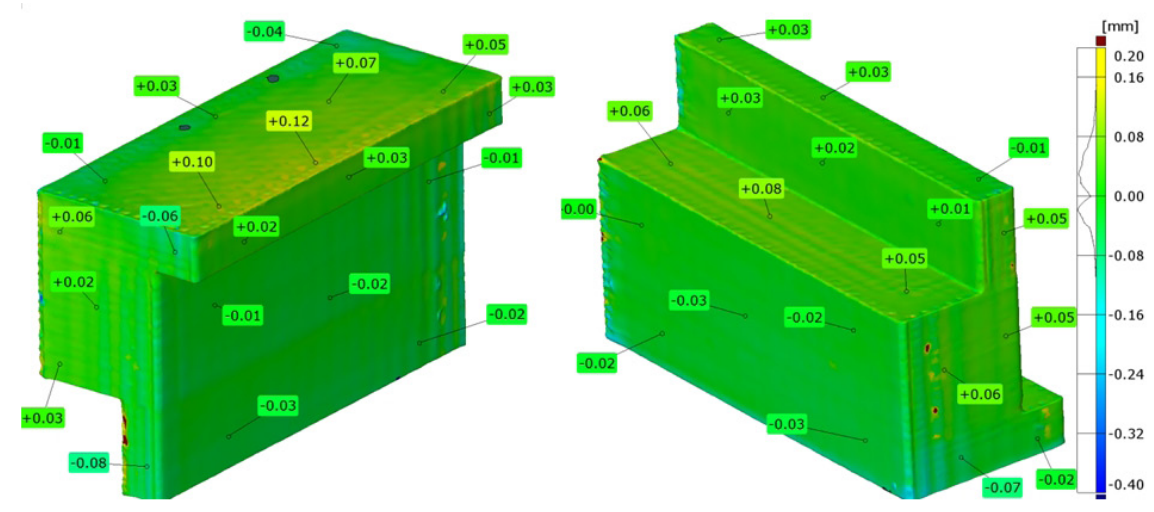

Fig. 6. Colorful map of deviations between a sample sterilized in plasma in horizontal orientation and the reference sample 

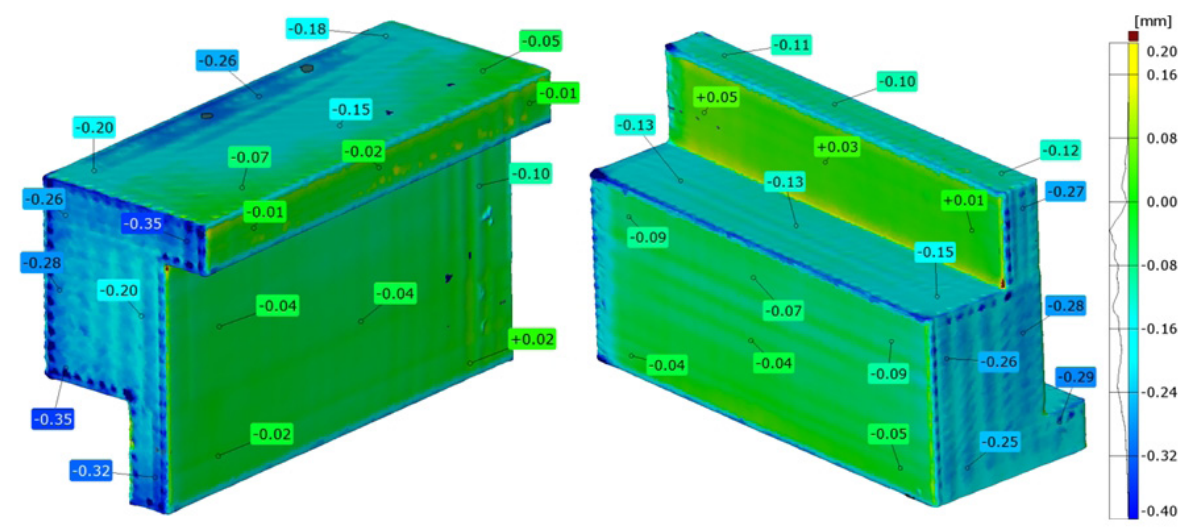

Fig. 7. Colorful map of deviations between a sample sterilized in plasma in vertical orientation and the reference sample

of samples in the plasma sterilization chamber brought different results in terms of accuracy the deviations were acceptable, but not negligible. Therefore, it cannot be said that the plasma sterilization process does not influence shape of a sterilized product at all. For more complex geometries, especially containing large surfaces of low thickness, the sterilization process can have bigger impact on the product accuracy, eliminating it from proper use. Evaluation of usefulness is obviously dependent on a specific application (type of surgery and function of a specific inter-operational supply), so it requires good communication and understanding between biomedical engineers and surgeons. In case of any doubts, procedure of measuring test samples should be performed.

\section{REFERENCES}

1. Bibb R., et. al.: Rapid manufacturing of customfitting surgical guides. Rapid Prototyping Journal, 2009, 15, 5, 346-354.

2. Chimento J., et al.: 3D printing tooling for thermoforming of medical devices. Rapid Prorotyping Journal, 2011, 17, 5, 387-392.

3. Gajdoš I., et al. Structure and tensile properties evaluation of samples produced by Fused Deposition Modeling. Open Engineering, 1(6), 2016, 86-89.

4. Górski F., et al.: Choosing optimal rapid manufacturing process for thin-walled products using expert algorithm. Journal of Industrial Engineering and Management, 2010, 3, 2, 408-420.

5. Górski F., et al.: Application of polystyrene pro- totypes manufactured by FDM technology for evaporative casting method. Modern Machinery Science Journal, 2017, 2, 1729-1733.

6. Górski F., et al.: Computation of mechanical properties of parts manufactured by fused deposition modeling using finite element method. Advances in Intelligent Systems and Computing, 2015, 368, 403-413.

7. Jardini A.L., et al.: Cranial reconstruction: $3 \mathrm{D}$ biomodel and custom-build implant created using additive manufacturing. Journal of Cranio-MaxilloFacial Surgery, 2014, 42, 8, 1877-1884.

8. O'Malley F.L., et al.: The use of adenosine triphosphate bioluminescence for assessing the cleanliness of additive-manufacturing materials used in medical applications. Additive Manufacturing, 2016, 9, 25-29.

9. Perez M., et al.: Sterilization of FDM-manufactured parts. 23rd Annual International Solid Freeform Fabrication Symposium - An Additive Manufacturing Conference, 2012, 285-296.

10. Popescu D., et al.: Low-temperature sterilization influence on Fused Deposition Modelling parts. Proceedings in Manufacturing Systems, 2016, 11, 3, 151-158.

11. Salmi M., et al.: Patient-specific reconstruction with 3D modelling and DMLS additive manufacturing. Rapid Prototyping Journal, 2012, 8, 3, 209-214.

12. Singare S., et al.: Rapid prototyping assisted surgery planning and custom implant design. Rapid Prototyping Journal, 2009, 15, 1, 19-23.

13. Tuomi J., et al.: A novel classification and online platform for planning and documentation of medical applications of additive manufacturing. Surgical Innovation, 2014, 21, 6, 553-559. 